\title{
Article \\ Photoredox-Catalyzed Giese Reactions: Decarboxylative Additions to Cyclic Vinylogous Amides and Esters
}

\author{
Kevin Dykstra ${ }^{1}$, Alexei Buevich ${ }^{2}$, Qi Gao ${ }^{2}$, Yu-Hong Lam ${ }^{3}$ and Jeffrey T. Kuethe ${ }^{4, *}$ \\ 1 Department of Medicinal Chemistry, Merck \& Co., Inc., 2000 Galloping Hill Road, Kenilworth, NJ 07033, USA; \\ Kevin_Dykstra@merck.com \\ 2 Analytical Research and Development, Merck \& Co., Inc., 2000 Galloping Hill Road, \\ Kenilworth, NJ 07033, USA; Alexei.Buevich@merck.com (A.B.); Qi.Gao1@merck.com (Q.G.) \\ 3 Department of Computational and Structural Chemistry, Merck \& Co., Inc., Rahway, NJ 07065, USA; \\ Yu.Hong.Lam@merck.com \\ 4 Department of Process Research and Development, Merck \& Co., Inc. Rahway, NJ 07065, USA \\ * Correspondence: Jeffrey_Kuethe@merck.com
}

\begin{abstract}
An effective strategy has been developed for the photoredox-catalyzed decarboxylative addition of cyclic amino acids to both vinylogous amides and esters leading to uniquely substituted heterocycles. The additions take place exclusively trans to the substituent present on the dihydropyridone ring affording stereochemical control about the new carbon-carbon bond. These reactions are operationally simplistic and afford the desired products in good to excellent isolated yields.
\end{abstract}

Keywords: photoredox; dihydropyridones; vinylogous amides and esters

check for

updates

Citation: Dykstra, K.; Buevich, A.; Gao, Q.; Lam, Y.-H.; Kuethe, J.T.

Photoredox-Catalyzed Giese Reactions: Decarboxylative Additions to Cyclic Vinylogous Amides and Esters. Molecules 2022, 27, 417. https://doi.org/10.3390/ molecules 27020417

Academic Editors: Damian Winston Young and Frederick A. Luzzio

Received: 29 November 2021 Accepted: 22 December 2021 Published: 10 January 2022

Publisher's Note: MDPI stays neutral with regard to jurisdictional claims in published maps and institutional affiliations.

Copyright: (c) 2022 by the authors. Licensee MDPI, Basel, Switzerland. This article is an open access article distributed under the terms and conditions of the Creative Commons Attribution (CC BY) license (https:// creativecommons.org/licenses/by/ $4.0 /)$.

\section{Introduction}

Reductive addition of carbon-centered radicals to electron-deficient olefins, known as the Giese reaction, has been utilized in a number of synthetic applications including total syntheses [1-7]. Visible-light photoredox-catalyzed Giese reactions have garnered a great deal of interest in recent years as a valuable method for the construction of carbon-carbon bonds in an atom-economical manner under relatively mild reaction conditions. Recent examples of carbon-centered radicals utilized in the Giese reaction have been generated via carboxylic acids [8-10], trifluoroborate salts [11,12], secondary and tertiary alcohols [13-15], organosilicates [16-18], alkyl halides [19], and via triplet enone diradicals [20]. Decarboxylative Giese reactions involving readily available amino acids have emerged as a powerful method for the construction of carbon-carbon bonds leading to the formation of molecules not previously accessible by other methods [8,9]. Reactions leading to increasing molecular complexity are extremely valuable synthetic tools and our interest in this area was inspired by the unique reactivity of cyclic vinylogous amides and esters of type 3 and 4 (Figure 1). It was envisioned that addition of carbon-centered radicals generated via a photocatalytic decarboxylation of amino acids would allow for the preparation of unprecedented and novel heterocyclic scaffolds 3 and 4 . In this manuscript we document our investigations in this area.<smiles>[R]OC(=O)C1C(C)C(=O)C=CN1CC</smiles>

1<smiles>CCC1OC=CC(=O)C1C</smiles>

2

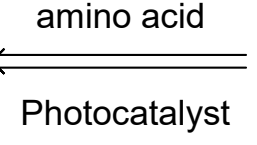

Photocatalyst<smiles>[X]C1C(CC)CC(=O)C(CC)C1C1CCN1[R]</smiles>

$$
3 \mathrm{X}=\mathrm{NCO}_{2} \mathrm{R}
$$$$
4 \mathrm{X}=0
$$

Figure 1. Decarboxylative additions to cyclic vinylogous amides and esters. 


\section{Results and Discussion}

Our investigations began by examining the reaction between dihydropyridone 5 [21] and amino acid 6 (Scheme 1). A high throughput screen of 24 photocatalysts was conducted employing potassium phosphate dibasic or potassium carbonate as bases and dimtheylformamide (DMF) or dimethylsulfoxide (DMSO) as solvents. The starting materials were dosed into pre-assembled vials containing a separate photocatalyst and base and were irradiated at $450 \mathrm{~nm}$ for $24 \mathrm{~h}$. The samples were then analyzed by liquid chromatography mass spectrometry (LCMS). The first screen yielded two "hits" for the desired mass. The photocatalysts of interest were identified as $\left(\operatorname{Ir}\left[\mathrm{dF}\left(\mathrm{CF}_{3}\right) \mathrm{ppy}_{2}(\mathrm{dtbby})\right) \mathrm{PF}_{6}[22]\right.$ and 1,2,3,5tetrakis(carbazol-9-yl)-4,6-dicyanobenzene (4CzIPN) [23] employing potassium phosphate dibasic and DMF. These reactions were subsequently scaled up to $100 \mathrm{mg}$ scale and purified by mass-directed HPLC. Interestingly, upon examining the nuclear magnetic resonance (NMR) of the isolated product it was found that the radical added exclusively trans to the phenyl group on the dihydropyridone. We speculate that the initially formed product 7 undergoes cyclization to aminal 8 during the mass-directed purification which employed aqueous trifluoroacetic acid (TFA) and acetonitrile. The observation that the radical approached trans to the phenyl group was further supported by molecular calculations which demonstrated that a cis approach is $3.8 \mathrm{kcal} / \mathrm{mol}$ higher in energy than the trans approach. This is perfectly in line with the fact that substituents on dihydropyridines bearing a carbamate prefer an axial orientation due to $\mathrm{A}^{1,3}$ strain between an equatorial substituent and the carbamate. This is also in line with the fact that Grignard reagents favor the trans products when reacted with dihydropyridones of type 5 . The resulting steric environment of the axial substituent leads to the radical approaching from the opposite face leading to the observed cis-isomer. Although the desired product was formed in the reaction, the isolated yield was $<10 \%$ for the iridium photocatalyst and $<5 \%$ for the 4 CzIPN catalyst. Reexamination of the crude reaction mixture revealed that at least two other major products had formed but were inseparable from one another. After a series of NMR experiments on the crude reaction mixture the structures were determined to be a 2:1 mixture of $2+2$ dimers 9 and 10. It is believed that the initially formed radical generated from amino acid 6 is sufficiently stable or self-quenches and dimerization becomes the major pathway. We speculate that intermolecular photocycloaddition leading to compounds $\mathbf{9}$ and $\mathbf{1 0}$ occurs through energy transfer from the iridium catalyst upon light absorption. The reaction was repeated with several other primary, secondary and tertiary acyclic amino acids and similar results were obtained.

Our attention then turned to screening cyclic amino acids in order to probe whether these would have better reactivity and deliver the desired product in useful yields. The initial photocatalytic screen was performed employing dihydropyridone 5 and Boc Lproline 11 (Scheme 2). The same optimal conditions discovered above were found also in this screen and the desired product was formed in much higher apparent yield. The optimal conditions employed were to irradiate at $450 \mathrm{~nm}$ the dihydropyridone 5 in DMF in the presence of $1 \mathrm{~mol} \% \operatorname{Ir}\left[\mathrm{dF}\left(\mathrm{CF}_{3}\right) \text { ppy }\right]_{2}$ (dtbby) and 2 equiv of both $\mathrm{K}_{2} \mathrm{HPO}_{4}$ and Boc L-proline for $20 \mathrm{~h}$. Under these conditions, the desired product 12 was obtained in $82 \%$ isolated yield and as a 1:1 inseparable mixture of diastereomers about the carbon where the radical was formed. There were only trace amounts of $2+2$ dimers in the crude NMR and these were easily separable from the products In addition, the trans product was the exclusive product formed, there being no detectable amounts of the cis products formed in the reaction. The trans stereochemistry was further confirmed by NMR. 
<smiles>O=C1C=CN(C(=O)Oc2ccccc2)C(c2ccccc2)C1</smiles>

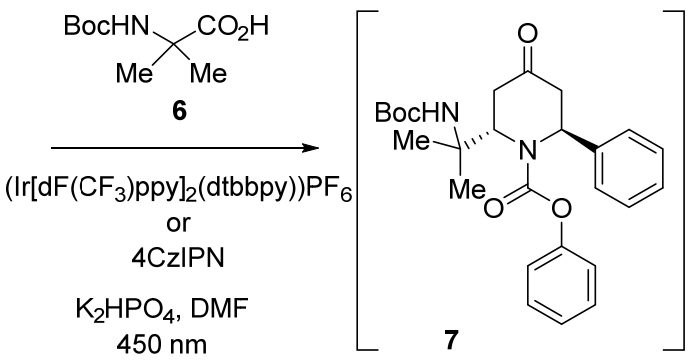<smiles>CC(C)(C)OC(=O)N1C2(O)CC(c3ccccc3)N(C(=O)Oc3ccccc3)C(C2)C1(C)C</smiles><smiles>O=C1C[C@H](c2ccccc2)[C@H]2[C@H]1[C@H]1C(=O)C[C@@H](c3ccccc3)N(C(=O)Oc3ccccc3)[C@H]21</smiles>
2:1<smiles>O=C1C[C@H](c2ccccc2)N(C(=O)Oc2ccccc2)[C@@H]2[C@@H]1[C@H]1C(=O)C[C@H](c3ccccc3)N(C(=O)Oc3ccccc3)[C@@H]12</smiles>

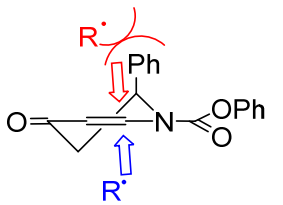

Scheme 1. Initial screening and observations.<smiles>O=C1C=CN(C(=O)Oc2ccccc2)C(c2ccccc2)C1</smiles>

5
$1 \mathrm{~mol} \%\left(\operatorname{Ir}\left[\mathrm{dF}\left(\mathrm{CF}_{3}\right) \mathrm{ppy}\right)_{2}(\mathrm{dtbpy})\right) \mathrm{PF}_{6}$

DMF, 2 equiv $\mathrm{K}_{2} \mathrm{HPO}_{4}$

PennOC Photoreactor, $450 \mathrm{~nm}$<smiles>O=C(O)C1CCCN1C(=O)O</smiles>

11<smiles>CC(C)(C)OC(=O)N1CCC[C@H]1[C@@H]1CC(=O)C[C@H](c2ccccc2)N1C(=O)Oc1ccccc1</smiles>

$1282 \%$

Scheme 2. Photocatalytic decoarboxyative addition of cyclic amino acids.

With these results in hand, the scope of the transformation was explored employing the optimized conditions. For example, an azetidine radical generated from Boc-protected amino acid 13 cleanly added to dihydropyridone 5 to give product $\mathbf{1 4}$ in $90 \%$ isolated yield and as an inseparable mixture of diastereomers. (Table 1, entry 1). Interestingly, reaction with amino acid 15 provided the desired product with modest levels of diastereoselectivity (2.8:1) where the major product of $\mathbf{1 6}$ could be isolated by fractional crystallization of the mixture from EtOAc/hexane after purification by silica gel chromatography (Table 1, entry 2). In similar fashion, reaction of amino acid 17 with dihydropyridone 5 also provided a 2.8:1 mixture of diastereomeric products 18; however, these products could not be separated from one another by either silica gel or fractional crystallization (Table 1, entry 3 ). The morpholine and indoline amino acids 19 and 21 also added to dihydropyridone 5 providing the desired products 20 and 22 in $85 \%$ and $69 \%$ yields, respectively (Table 1 , entries 4,5 ). In both cases, there were no observable levels of diastereoselectivety and a 1:1 mixture of products was obtained. The diastereomers of $\mathbf{2 0}$ were separable by silica gel chromatography; however, the diastereomers of 22 were inseparable. As an extension of these investigations, it was discovered that tetrahydropyran-2-carboxylic acid 23 could also be utilized leading to compound 24 in 58\% yield and a separable 2:1 mixture of diastereomers (Table 1, entry 6). In addition, dihydrobenzofuran-2-carboxylic acid 25 
underwent smooth decarboxylative radical formation and addition to dihydropyridone 5 to provide product $\mathbf{2 6}$ in $82 \%$ isolated yields and as a 3:1 separable mixture of diastereomers (Table 1, entry 7). The major diastereomer of compound 26 was subjected to a series of NMR experiments and density functional theory (DFT) calculations in order to determine the configuration of the 3 chiral centers of the major diastereomer. From these experiments it was determined that the major diastereomer 26a had the stereochemistry as depicted in Scheme 3.

Table 1. Scope of amino acid additions to dihydropyridone $\mathbf{5}$.

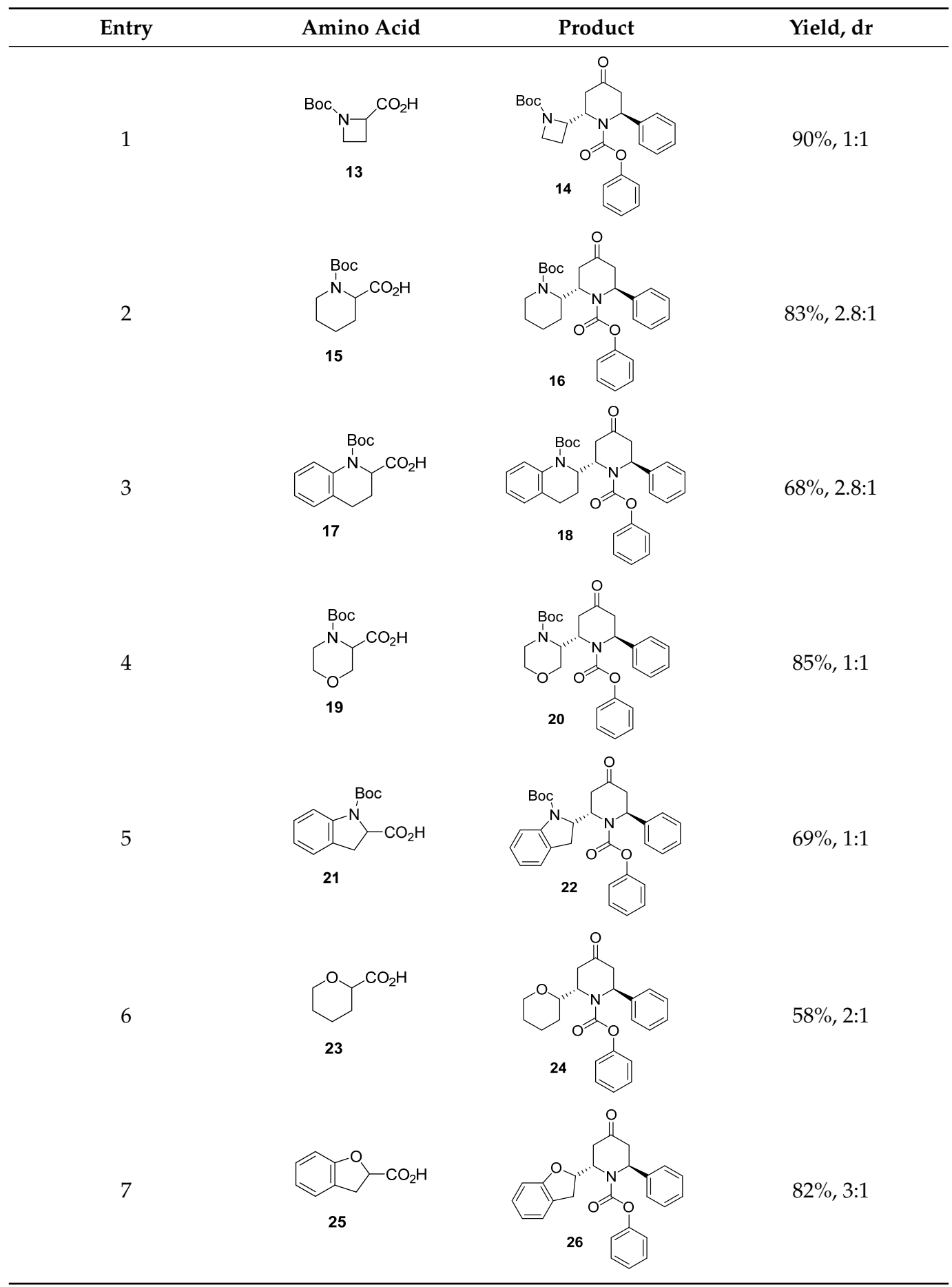



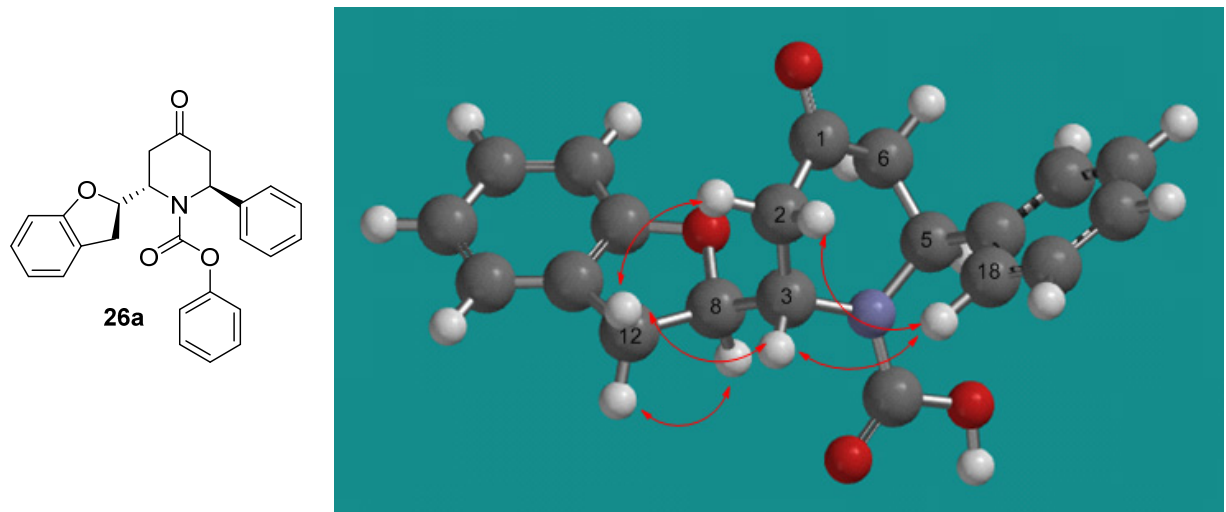

Scheme 3. Confirmed stereochemistry of major diastereomer of compound 26.

Reaction of tetrahydrofuran-2-carboxylic acid $\mathbf{2 7}$ with dihyropyridone 5 under the

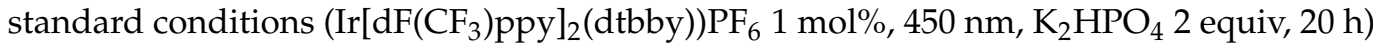
led to full conversion and afforded the expected product 28 as a separable 3:2 mixture of diasteremers and was isolated in $70 \%$ yield (Scheme 4 ). A complementary approach to this molecule involved the recently reported nickel-catalyzed addition of THF to enones involving an energy-transfer initiated catalysis involving triplet diradicals [20]. Irradiation at $450 \mathrm{~nm}$ of dihydropyridone 5 in the presence of $1 \mathrm{~mol} \%\left(\operatorname{Ir}\left[\mathrm{dF}_{(}\left(\mathrm{CF}_{3}\right) \text { ppy }\right]_{2}(\mathrm{dtbby})\right) \mathrm{PF}_{6}$ in the presence of $5 \mathrm{~mol}^{\%} \mathrm{NiBr}_{2}$ glyme, $15 \mathrm{~mol} \%$ 2,2'-bis(2-oxazoline (BiOx) in tetrahydrofuran (THF) resulted in $82 \%$ conversion after $24 \mathrm{~h}$ and afforded compound 28 in $70 \%$ isolated yield. Under these conditions, compound 28 was obtained as a 3:2 mixture of diastereomers which was identical to the decarboxylative process and was obtained in similar levels of diastereoselectivity.

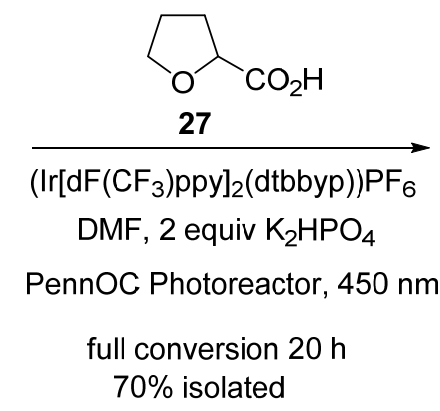

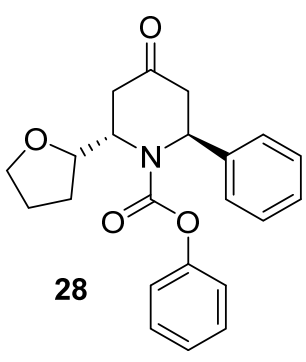

(3:2 diastereomers)

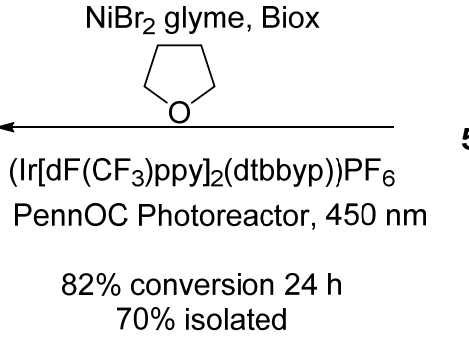

Scheme 4. Complementary additions of THF to dihydropyridone 5.

We next turned our attention to the addition of these carbon-centered radicals to both 4-oxoquinolines 29 [24] and chromen-4-ones 30 to further broaden the scope of the transformation (Scheme 5). As revealed in Table 2, reaction with amino acids $\mathbf{1 3}$ and $\mathbf{1 1}$ with 4-oxoquinoline $\mathbf{2 9}$ under the standard conditions provided products $\mathbf{3 1}$ and $\mathbf{3 2}$ as a 1:1 mixture of diastereomers in $38 \%$ and $55 \%$ isolated yields, respectively. The individual diastereomers of product 31 were separable whereas the diastereomers of product 32 could not be separated from one another. Reaction of compound 29 with amino acids 14 and 16 provided the desired products 33 and 34 in slightly higher yield. In each of these cases the observed diastereoselectivity increased to $4: 1$; however, these diastereomers were inseparable from one another. Reaction of chromenone $\mathbf{3 0}$ with the radicals generated from amino acids $\mathbf{1 1}$ and $\mathbf{2 1}$, was also successful providing the addition products $\mathbf{3 5}$ and 36 in good yields and as inseparable mixtures of diastereomers. In addition, the radical generated from tetrahydrofuran-2-carboxylic acid gave product 37 in $68 \%$ isolated yield where the individual diastereomers could be separated from each other. Finally, reaction of 
chromenone 30 with amino acid 15 afforded heterocycle 38 in 92\% yield and as a 1:1 mixture of separable diastereomers.<smiles>O=C(O)n1ccc(=O)c2ccccc21</smiles>

29<smiles>O=c1ccoc2ccccc12</smiles>

30

Scheme 5. 4-oxoquinoline 29 and chromen-4-one 30.

Table 2. Photocatalytic decarboxylative additions to 4-Oxoquinline 29 and chromenone $\mathbf{3 0 .}$

\begin{tabular}{|c|c|c|c|c|}
\hline Entry & $\begin{array}{l}\text { Starting } \\
\text { Material }\end{array}$ & Amino Acid & Product & Yield, dr \\
\hline 1 & 29 & 13 & 31 & $38 \%, 1: 1$ \\
\hline 2 & 29 & 11 & 32 & $55 \%, 1: 1$ \\
\hline 3 & 29 & 14 & 33 & $61 \%, 4: 1$ \\
\hline 4 & 29 & 16 & 34 & $65 \%, 4: 1$ \\
\hline 5 & 30 & 11 & & $65 \%, 1: 1$ \\
\hline 6 & 30 & 21 & & $61 \%, 1: 1$ \\
\hline 7 & 30 & 27 & & $68 \%, 1: 1$ \\
\hline 8 & 30 & 15 & & $92 \%, 1: 1$ \\
\hline
\end{tabular}




\section{Materials and Methods}

All anhydrous solvents were supplied by Sigma Aldrich in Sureseal ${ }^{\circledR}$ bottles and used without further purification. All commercially available chemicals were used as received. Reactions were monitored by ultra-performance liquid chromatography UPLC employing an Agilent Technologies 1290 Infinity II UPLC with a Waters Aquity UPLC DEH C18 column $\left(1.7 \mathrm{~mm}, 2.1 \times 100 \mathrm{~mm}, 0.4 \mathrm{~mL} / \mathrm{min}, 40{ }^{\circ} \mathrm{C}\right.$ solvent A $0.1 \% \mathrm{H}_{3} \mathrm{PO}_{4} /$ water: $\mathrm{B}$ $\mathrm{MeCN}, 90: 10$ to 10:90 A:B over $8 \mathrm{~min}$ ). Silica gel chromatography was performed with a 24-gram pre-packaged cartridge on a Teledyne ISCO CombiFlash Rf using a gradient of 0-100\% methyl tert-butyl ether (MTBE)/hexane. NMR spectra were obtained on a Bruker $500 \mathrm{MHz}$ spectrometer. Elemental analysis was performed at Intertek Pharmaceutical Services. HMRS were obtained at Merck \& Co., Inc., Kenilworth, NJ, USA.

Preparation of Trans-phenyl-1-(tert-butoxycarbonyl)pyrrolidin-2-yl)-4-oxo-6-phenylpiperidine-1carboxylate (12). According the the general procedure, reaction of $200 \mathrm{mg}(10.68 \mathrm{mmol})$ of dihydropyridone 5 with $294 \mathrm{mg}(1.36 \mathrm{mmol})$ of $\mathrm{N}$-Boc-L-proline 11 in the presence of

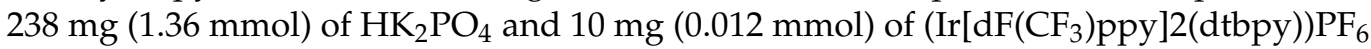
provided $260 \mathrm{mg}(82 \%)$ of compound 12 as a colorless oil and as inseparable 1:1 mixture of diastereomers and rotamers: ${ }^{1} \mathrm{H}$ NMR $\left(500 \mathrm{MHz}, \mathrm{CDCl}_{3}\right) \delta 7.36(\mathrm{~m}, 2 \mathrm{H}), 7.30-7.12(\mathrm{~m}$, $7 \mathrm{H}), 6.74(\mathrm{~d}, 2 \mathrm{H}, J=7.9 \mathrm{~Hz}), 5.73$ and $5.61(\mathrm{~d}, 1 \mathrm{H}, J=6.4$ and $6.6 \mathrm{~Hz}), 4.65-4.47(\mathrm{~m}, 1.17 \mathrm{H})$, $4.39-4.29(\mathrm{~m}, 0.64 \mathrm{H}), 3.97(\mathrm{~m}, 0.66 \mathrm{H}), 3.60-3.49(\mathrm{~m}, 0.80 \mathrm{H}), 3.47-3.31(\mathrm{~m}, 1 \mathrm{H}), 3.20(\mathrm{br} \mathrm{m}$, $0.30 \mathrm{H}), 2.85(\mathrm{~m}, 1.3 \mathrm{H}), 2.69-2.54(\mathrm{~m}, 1.27 \mathrm{H}), 2.23(\mathrm{~m}, 0.57 \mathrm{H}), 2.05-1.87(\mathrm{~m}, 2.6 \mathrm{H}), 1.68(\mathrm{~m}$, 0.62H), 1.63-1.45 (m, 9H); ${ }^{13} \mathrm{C}-\mathrm{NMR}\left(\mathrm{CDCl}_{3}, 125 \mathrm{MHz}\right) \delta 206.6,155.6,155.2,151.1,143.5$, $129.3,129.1,128.9,128.8,127.4,127.2,127.0,125.5,125.3,125.2,125.1,121.8,121.6,121.5,80.1$, 79.5, 60.9, 56.5, 56.2, 55.5, 54.5, 53.7, 47.4, 47.2, 46.1, 46.0, 44.8, 41.0, 40.9, 39.7, 29.1, 29.0, 28.6, 28.4, 27.0, 23.3, 23.0. HRMS Calcd. For $\mathrm{C}_{27} \mathrm{H}_{33} \mathrm{~N}_{2} \mathrm{O}_{5}[\mathrm{M}=\mathrm{H}$ ?: 465.2389. Found: 465.2380.

Preparation of Trans-1-(tert-butoxycarbonyl)azetidin-2-yl)-4-oxo-6-phenylpiperidine-1-carboxylate (14). According the the general procedure, reaction of $160 \mathrm{mg}(0.55 \mathrm{mmol})$ of dihydropyridone 5 with $220 \mathrm{mg}(1.10 \mathrm{mmol}) \mathrm{N}$-Boc-azetidine-2-carboxylic acid 13 in the presence of $190 \mathrm{mg}(1.13 \mathrm{mmol})$ of $\mathrm{HK}_{2} \mathrm{PO}_{4}$ and $9.4 \mathrm{mg}(8.4 \mathrm{mmol})$ of $\left(\operatorname{Ir}\left[\mathrm{dF}\left(\mathrm{CF}_{3}\right) \mathrm{ppy}\right] 2(\mathrm{dtbpy})\right) \mathrm{PF}_{6}$ provided $220 \mathrm{mg}(90 \%)$ of compound $\mathbf{1 4}$ as colorless oil and as an inseparable 1:1 mixture of diastereomers and rotamers: ${ }^{1} \mathrm{H}$ NMR $\left(500 \mathrm{MHz}, \mathrm{CDCl}_{3}\right) \delta 7.42-7.27(\mathrm{~m}, 7 \mathrm{H}), 7.19$ (br m, 2H), $6.95($ br m, 1H), $5.78(\mathrm{~m}, 1 \mathrm{H}), 5.04(\mathrm{~m}, 0.5 \mathrm{H}), 4.93(\mathrm{~m}, 0.5 \mathrm{H}), 4.60(\mathrm{~m}, 0.5 \mathrm{H}), 4.38$ $(\mathrm{m}, 0.5 \mathrm{H}), 3.91(\mathrm{~m}, 1.5 \mathrm{H}), 3.75(\mathrm{dt}, 1.5 \mathrm{H}, J=9.0$ and $6.7 \mathrm{~Hz}), 3.42(\mathrm{~m}, 0.5 \mathrm{H}), 2.99-2.81(\mathrm{~m}$, $2 \mathrm{H}), 2.72(\mathrm{~m}, 0.5 \mathrm{H}), 2.54(\mathrm{~m}, 1.5 \mathrm{H}), 1.94(\mathrm{~m}, 1 \mathrm{H}), 1.51$ and $1.49(\mathrm{~s}, 9 \mathrm{H}) ;{ }^{13} \mathrm{C}-\mathrm{NMR}\left(\mathrm{CDCl}_{3}\right.$, $125 \mathrm{MHz}) \delta 205.8$ and 205.1, 157.8, 151.0, 142.1, 129.4, 129.3, 129.2, 129.0, 128.9, 127.4, 127.3, $125.6,125.4,125.3,125.2,121.6,80.4,64.9,64.4,56.4,55.2,54.5,47.3,45.8,44.2,39.4,37.7,28.5$ and 28.4, 21.3, 20.0. HRMS Cacld. For $\mathrm{C}_{26} \mathrm{H}_{31} \mathrm{~N}_{2} \mathrm{O}_{5}[\mathrm{M}+\mathrm{H}]: 451.2233$. Found: 451.2239.

Preparation of Trans-1-(tert-butyl-1-phenyl-4-oxo-6-phenyl-[2,2'-bipiperidine]-1,1'dicarboxylate (16). According the the general procedure, reaction of $133 \mathrm{mg}(0.45 \mathrm{mmol})$ of dihydropyridone 5 with $158 \mathrm{mg}(0.91 \mathrm{mmol}) \mathrm{N}$-Boc-pipecolic acid 15 in the presence of $208 \mathrm{mg}$ $(0.91 \mathrm{mmol})$ of $\mathrm{HK}_{2} \mathrm{PO}_{4}$ and $7.5 \mathrm{mg}(6.7 \mathrm{mmol})$ of $\left(\mathrm{Ir}\left[\mathrm{dF}\left(\mathrm{CF}_{3}\right) \mathrm{ppy}\right] 2(\mathrm{dtbpy})\right) \mathrm{PF}_{6}$ provided $183 \mathrm{mg}(83 \%)$ of compound $\mathbf{1 6}$ as separable 2.8:1 mixture of diastereomers and rotamers: First Isomer to elute: colorless oil; ${ }^{1} \mathrm{H}$ NMR $\left(500 \mathrm{MHz}, \mathrm{CDCl}_{3}\right) \delta 7.39(\mathrm{~m}, 2 \mathrm{H}), 7.24(\mathrm{~m}$, $5 \mathrm{H}), 7.13(\mathrm{~m}, 1 \mathrm{H}), 6.62(\mathrm{br} \mathrm{m}, 2 \mathrm{H}), 6.66$ and $5.52(\mathrm{br} \mathrm{m}, 1 \mathrm{H}), 5.18$ and $5.07(\mathrm{br} \mathrm{m}, 1 \mathrm{H}), 4.42$ (br m, 1H), 4.16 (br m, 1H), $3.99(\mathrm{~d}, 1 \mathrm{H}, J=12.4 \mathrm{~Hz}), 3.55-3.32($ br m, 1H), 2.89-2.55 (m, 2H), 1.74-1.48 (m, 15H);); ${ }^{13} \mathrm{C}-\mathrm{NMR}\left(\mathrm{CDCl}_{3}, 125 \mathrm{MHz}\right) \delta 206.2,158.9,129.1,129.0,128.9,127.4$, 127.2, 125.5, 125.4, 125.0, 121.5, 121.4, 56.8, 53.6, 49.9, 45.5, 41.3, 40.7, 28.5, 28.1, 25.6, 24.8, 18.9. HRMS calcd for $\mathrm{C}_{28} \mathrm{H}_{34} \mathrm{~N}_{2} \mathrm{O}_{5}$ [M + H]: 479.2546. Found: 479.2552. Second Isomer to elute: colorless solid; ${ }^{1} \mathrm{H}$ NMR $\left(500 \mathrm{MHz}, \mathrm{CDCl}_{3}\right) \delta 7.50(\mathrm{~m}, 2 \mathrm{H}), 7.25(\mathrm{~m}, 5 \mathrm{H}), 7.16(\mathrm{~m}, 1 \mathrm{H})$, $6.74(\mathrm{br} \mathrm{m}, 2 \mathrm{H}), 5.66(\mathrm{~m}, 1 \mathrm{H}), 5.11(\mathrm{~m}, 1 \mathrm{H}), 4.70(\mathrm{br} \mathrm{m}, 0.5 \mathrm{H}), 4.50(\mathrm{br} \mathrm{m}, 0.5 \mathrm{H}), 4.21(\mathrm{~d}, 0.5 \mathrm{H}$, $J=12.7 \mathrm{~Hz}), 4.01(\mathrm{~d}, 0.5 \mathrm{H}, J=12.4 \mathrm{~Hz}), 3.69(\mathrm{~m}, 0.5 \mathrm{H}), 3.43(\mathrm{~m}, 0.5 \mathrm{H}), 2.89-2.54(\mathrm{~m}, 4 \mathrm{H})$, 1.94 (br m, 2H), 1.72-1.53 (m, 3H), $1.46(\mathrm{~s}, 9 \mathrm{H}) ;{ }^{13} \mathrm{C}-\mathrm{NMR}\left(\mathrm{CDCl}_{3}, 125 \mathrm{MHz}\right) \delta 204.3,155.3$, $145.3,150.8,129.3,129.2$, 129.0, 128.9, 127.6, 127.4, 125.7, 125.6, 125.0, 121.4, 80.9, 80.2, 56.4, $56.3,54.8,53.2,49.8,49.4,45.4,45.2,40.6,39.7,39.6,39.0,28.5,28.4,28.2,25.7,25.3,25.0,24.7$, 
19.4, 19.3, 18.9. Anal. Calcd. For $\mathrm{C}_{28} \mathrm{H}_{34} \mathrm{~N}_{2} \mathrm{O}_{5}$ : C, 70.27; $\mathrm{H}, 7.16 ; \mathrm{N}, 5.85$. Found, $\mathrm{C}, 69.97 ; \mathrm{H}$, $6.99 ; \mathrm{N}, 5.82$.

Preparation of Trans-tert-butyl-4-oxo-1-(phenoxycarbonyl)-6-phenylpiperidin-2-yl)-3,4dihydroquinoline-1(2H)-carboxylate (18). According the the general procedure, reaction of $164 \mathrm{mg}(0.56 \mathrm{mmol})$ of dihydropyridone 5 with $310 \mathrm{mg}(1.12 \mathrm{mmol})$ of $\mathrm{N}$-Boctetrahydroquinoline-2-carboxylic acid 17 in the presence of $195 \mathrm{mg}(1.12 \mathrm{mmol})$ of $\mathrm{HK}_{2} \mathrm{PO}_{4}$ and $13 \mathrm{mg}(0.011 \mathrm{mmol})$ of $\left(\operatorname{Ir}\left[\mathrm{dF}\left(\mathrm{CF}_{3}\right)\right.\right.$ ppy $\left.] 2(\mathrm{dtbpy})\right) \mathrm{PF}_{6}$ provided $202 \mathrm{mg}(68 \%)$ of compound 18 as colorless oil and an inseparable 2.8:1 mixture of diastereomers and rotamers: ${ }^{1} \mathrm{H} \mathrm{NMR}\left(500 \mathrm{MHz}, \mathrm{CDCl}_{3}\right) \delta 7.45-7.40(\mathrm{~m}, 12 \mathrm{H}), 6.75$ and $6.88(\mathrm{br} \mathrm{m}, 2 \mathrm{H}), 5.79$ and $5.73(\mathrm{~d}$, $1 \mathrm{H}, J=5.6$ and $6.9 \mathrm{~Hz}), 5.11$ and $4.84(\mathrm{~m}, 1 \mathrm{H}), 4.64-4.57(\mathrm{br} \mathrm{m}, 1 \mathrm{H}), 4.03$ and $3.57(\mathrm{~m}, 1 \mathrm{H})$, 3.01-2.89 $(\mathrm{m}, 2 \mathrm{H}), 2.57-2.38(\mathrm{~m}, 3 \mathrm{H}), 2.00(\mathrm{~m}, 1 \mathrm{H}), 1.54$ and $1.51(\mathrm{~s}, 9 \mathrm{H}) ;{ }^{13} \mathrm{C}-\mathrm{NMR}\left(\mathrm{CDCl}_{3}\right.$, $125 \mathrm{MHz}) \delta 206.1$ and 204.6, 155.1 and 154.7, 151.2 and 150.9, 143.8 and 142.2, 137.1 and $136.3,131.6,129.4,129.1,128.9,128.8,128.4$ and $128.3,127.5$ and 127.3, 126.2, 126.1, 125.9, $125.7,125.2,125.1,124.8,124.7,121.6$ and $121.4,81.7$ and 81.1, 57.0, 56.5, 55.5, 54.9, 45.7 and 45.0, 41.1, 39.3, 30.3, 28.4 and 28.3, 26.9, 26.2, 24.2. HRMS Calcd. For $\mathrm{C}_{32} \mathrm{H}_{35} \mathrm{~N}_{2} \mathrm{O}_{5}[\mathrm{M}+\mathrm{H}]$ : 527.2546 Found: 527.2553.

Preparation of Trans-tert-butyl-4-oxo-1-(phenoxycarbonyl)-6-phenylpiperidin-2-yl)morpholine-4carboxylate (20). According the the general procedure, reaction of $158 \mathrm{mg}(0.539 \mathrm{mmol})$ of dihydropyridone 5 with $249 \mathrm{mg}(1.08 \mathrm{mmol})$ of 4-Boc-3-morpholinecarboxylic acid 19 in the presence of $188 \mathrm{mg}(1.08 \mathrm{mmol})$ of $\mathrm{HK}_{2} \mathrm{PO}_{4}$ and $9.1 \mathrm{mg}(8.1 \mathrm{mmol})$ of $\left(\operatorname{Ir}\left[\mathrm{dF}\left(\mathrm{CF}_{3}\right) \mathrm{ppy}\right] 2\right.$ (dtbpy)) $\mathrm{PF}_{6}$ provided $310 \mathrm{mg}(65 \%)$ of compound 20 as a separable 1:1 mixture of diastereomers and rotamers in 85\% combined yield. Isomer \#1: colorless oil; ${ }^{1} \mathrm{H}$ NMR $(500 \mathrm{MHz}$, $\left.\mathrm{CDCl}_{3}\right) \delta 7.45-7.24(\mathrm{~m}, 7 \mathrm{H}), 7.14(\mathrm{~m}, 1 \mathrm{H}), 6.62(\mathrm{br} \mathrm{m}, 2 \mathrm{H}), 5.65$ and $5.51(\mathrm{~d}, 1 \mathrm{H}, J=6.8$ and 7.2 Hz), 5.40-5.31 (m, 1H), 4.15-3.47 (m, 7H), 3.10-3.02 (m, 1H), 2.93-2.75 (m, 2H), 1.61 and $1.54(\mathrm{~m}, 9 \mathrm{H}), 1.50(\mathrm{~m}, 1 \mathrm{H}) ;{ }^{13} \mathrm{C}-\mathrm{NMR}\left(\mathrm{CDCl}_{3}, 125 \mathrm{MHz}\right) \delta$ 206.0, 205.0, 155.5, 150.9, $143.9,129.3,129.1,129.0,128.9,127.6,127.3,125.7,125.5,125.1,125.0,121.8,121.5,80.9$, 80.6, 67.5, 66.9, 66.6, 57.0, 56.2, 55.9, 53.9, 48.6, 45.5, 44.9, 41.1, 40.8, 39.3, 28.7, 28.5, 28.3, 28.1. HRMS Calcd. For $\mathrm{C}_{27} \mathrm{H}_{33} \mathrm{~N}_{2} \mathrm{O}_{5}$ [M $\left.+\mathrm{H}\right]$ : 480.2260. Found: 480.2257. Isomer \#2: colorless oil; ${ }^{1} \mathrm{H}$ NMR $\left(500 \mathrm{MHz}, \mathrm{CDCl}_{3}\right) \delta 7.39(\mathrm{~m}, 2 \mathrm{H}), 7.28(\mathrm{~m}, 5 \mathrm{H}), 7.16(\mathrm{~m}, 1 \mathrm{H}), 6.87$ (br m, 2H), $5.69(\mathrm{~m}, 1 \mathrm{H}), 5.27(\mathrm{~m}, 1 \mathrm{H}), 4.48(\mathrm{~d}, 0.5 \mathrm{H}, J=7.9 \mathrm{~Hz}), 4.30(\mathrm{~d}, 0.5 \mathrm{H}, J=8.2 \mathrm{~Hz})$, $4.20(\mathrm{~d}, 0.5 \mathrm{H}, J=12.0 \mathrm{~Hz}), 4.13(\mathrm{~d}, 0.5 \mathrm{H}, J=12.0 \mathrm{~Hz}), 4.03(\mathrm{~m}, 0.5 \mathrm{H}), 3.90(\mathrm{~m}, 1 \mathrm{H}), 3.81(\mathrm{~d}$, $0.5 \mathrm{H}, J=12.5 \mathrm{~Hz}), 3.64(\mathrm{~m}, 1.7 \mathrm{H}), 3.50(\mathrm{~m}, 1.3 \mathrm{H}), 3.33(\mathrm{dd}, 0.5 \mathrm{H}, J=16.9$ and $6.9 \mathrm{~Hz}), 3.09$ $(\mathrm{m}, 0.5 \mathrm{H}), 3.00-2.69(\mathrm{~m}, 3 \mathrm{H}), 2.60(\mathrm{~m}, 0.5 \mathrm{H}), 1.51(\mathrm{~m}, 1 \mathrm{H}), 1.48(\mathrm{~s}, 9 \mathrm{H})$. HRMS Calcd. For $\mathrm{C}_{27} \mathrm{H}_{33} \mathrm{~N}_{2} \mathrm{O}_{5}[\mathrm{M}+\mathrm{H}]: 481.2339$. Found: 481.2242 .

Preparation of Trans-tert-butyl-4-oxo-1-(phenoxycarbonyl)-6-phenylpiperidine-2-yl)indoline-1carboxylate (22). According the the general procedure, reaction of $166 \mathrm{mg}(0.56 \mathrm{mmol})$ of pyridone 5 with $298 \mathrm{mg}(1.13 \mathrm{mmol}) \mathrm{N}$-Boc-indoline-2-carboxylic acid 21 in the presence of $197 \mathrm{mg}(1.13 \mathrm{mmol})$ of $\mathrm{HK}_{2} \mathrm{PO}_{4}$ and $9.4 \mathrm{mg}(8.4 \mathrm{mmol})$ of $\left(\mathrm{Ir}\left[\mathrm{dF}\left(\mathrm{CF}_{3}\right)\right.\right.$ ppy $\left.] 2(\mathrm{dtbpy})\right) \mathrm{PF}_{6}$ provided $201 \mathrm{mg}(69 \%)$ of compound 22 as colorless oil and as an inseparable 1:1 mixture of diastereomers and rotamers: ${ }^{1} \mathrm{H}$ NMR $\left(500 \mathrm{MHz}, \mathrm{CDCl}_{3}\right) \delta 7.49-6.70(\mathrm{~m}, 15 \mathrm{H}), 5.91$ and $5.82(\mathrm{~d}, 1 \mathrm{H}, J=7.5$ and $6.5 \mathrm{~Hz}), 5.62(\mathrm{br} \mathrm{m}, 0.5 \mathrm{H}), 5.45(\mathrm{br} \mathrm{m}, 1 \mathrm{H}), 4.66(\mathrm{~m}, 1 \mathrm{H}), 4.57(\mathrm{~m}$, $1 \mathrm{H}), 3.55(\mathrm{dd}, 1 \mathrm{H}, J=16.5$ and $10.1 \mathrm{~Hz}), 3.41(\mathrm{dd}, 0.5 \mathrm{H}, J=16.0$ and $8.3 \mathrm{~Hz}), 3.29(\mathrm{dd}, 1.2 \mathrm{H}$, $J=16.8$ and $7.4 \mathrm{H}), 2.99-2.90(\mathrm{br} \mathrm{m}, 2.3 \mathrm{H}), 2.73(\mathrm{br} \mathrm{dd}, 2.25 \mathrm{H}, J=29.2$ and $17.5 \mathrm{~Hz}), 2.49$ $(\mathrm{dd}, 1.2 \mathrm{H}, J=17.5$ and $7.3 \mathrm{~Hz}), 2.23(\mathrm{~d}, 1 \mathrm{H}, J=17.8 \mathrm{~Hz}), 1.65$ and $1.63(\mathrm{~s}, 9 \mathrm{H}) ;{ }^{13} \mathrm{C}-\mathrm{NMR}$ $\left(\mathrm{CDCl}_{3}, 125 \mathrm{MHz}\right) \delta 206.4,203.5,153.5,152.5,151.2,142.6,142.5,130.4,129.6,129.5,129.3$, $129.1,128.9,128.3,127.7,127.4,125.6,125.3,125.1,124.5,123.8,123.2,121.7,121.3,82.0,62.5$, 60.3, 58.6, 57.5, 56.7, 51.7, 46.1, 44.0, 43.1, 40.1, 37.8, 34.8, 32.6, 28.5, 28.4. HRMS Calcd. For $\mathrm{C}_{31} \mathrm{H}_{33} \mathrm{~N}_{2} \mathrm{O}_{5}[\mathrm{M}+\mathrm{H}]: 513.2389$. Found: 513.2379.

Preparation of Trans-phenyl-4-oxo-2-phenyl-2-tetrahydro-2H-pyran-2-yl)piperidine-1-carboxylate (24). According the the general procedure, reaction of $160 \mathrm{mg}(0.55 \mathrm{mmol})$ of dihydropyridone 5 with $142 \mathrm{mg}(1.09 \mathrm{mmol})$ tetrahydropyran-2-carboxylic acid 23 in the presence of $190 \mathrm{mg}(1.09 \mathrm{mmol})$ of $\mathrm{HK}_{2} \mathrm{PO}_{4}$ and $6.0 \mathrm{mg}(8.2 \mathrm{mmol})$ of $\left(\operatorname{Ir}\left[\mathrm{dF}\left(\mathrm{CF}_{3}\right) \mathrm{ppy}\right] 2(\mathrm{dtbpy})\right) \mathrm{PF}_{6}$ 
provided $120 \mathrm{mg}$ (58\%) of compound 24 as a separable 2:1 mixture of diastereomers: Major Isomer: colorless oil; ${ }^{1} \mathrm{H}$ NMR $\left(500 \mathrm{MHz}, \mathrm{CDCl}_{3}\right) \delta 7.45-7.21(\mathrm{~m}, 8.5 \mathrm{H}), 6.99$ (br m, 1.5H), $5.80(\mathrm{~s}, 1 \mathrm{H}), 4.44(\mathrm{~s}, 1 \mathrm{H}), 4.00(\mathrm{~d}, 1 \mathrm{H}, J=10.8 \mathrm{~Hz}), 3.90(\mathrm{~d}, 1 \mathrm{H}, J=11.5 \mathrm{~Hz}), 3.61(\mathrm{~m}, 1 \mathrm{H}), 3.44$ $(\mathrm{m}, 1 \mathrm{H}), 2.95(\mathrm{dd}, 1 \mathrm{H}, J=17.4$ and $1.8 \mathrm{~Hz}), 2.67(\mathrm{~d}, 1 \mathrm{H}, J=17.4 \mathrm{~Hz}), 2.49(\mathrm{br} \mathrm{dd}, 1 \mathrm{H}, J=17.4$ and 7.1 Hz), $1.89(\mathrm{~m}, 1 \mathrm{H}), 1.66-1.49(\mathrm{~m}, 4 \mathrm{H}), 1.34(\mathrm{~m}, 1 \mathrm{H}) ;{ }^{13} \mathrm{C}-\mathrm{NMR}\left(\mathrm{CDCl}_{3}, 125 \mathrm{MHz}\right)$ $\delta$ 206.1, 155.1, 151.0, 142.1, 129.3, 128.9, 127.3, 125.6, 125.3, 121.7, 79.4, 68.5, 57.0, 54.4, 45.1, 37.5, 28.3, 25.6, 23.4. HRMS Calcd. For $\mathrm{C}_{23} \mathrm{H}_{26} \mathrm{NO}_{4}[\mathrm{M}+\mathrm{H}]: 379.1784$. Found: 379.1784 . Minor Isomer: colorless oil; ${ }^{1} \mathrm{H}$ NMR $\left(500 \mathrm{MHz}, \mathrm{CDCl}_{3}\right) \delta 7.38(\mathrm{t}, 2 \mathrm{H}, J=7.6 \mathrm{~Hz}), 7.26(\mathrm{~m}$, $5 \mathrm{H}), 7.17(\mathrm{t}, 1 \mathrm{H}, J=7.3 \mathrm{~Hz}), 6.83(\mathrm{br} \mathrm{m}, 2 \mathrm{H}), 5.74(\mathrm{~d}, 1 \mathrm{H}, J=5.7 \mathrm{~Hz}), 4.87(\mathrm{~m}, 1 \mathrm{H}), 4.04(\mathrm{~d}$, $1 \mathrm{H}, J=8.5 \mathrm{~Hz}), 3.65$ (br m, 1H), 3.50-3.39 (m, 2H), 2.85-2.76 (m, 2H), 2.61 (dd, $1 \mathrm{H}, J=17.7$ and $1.5 \mathrm{~Hz}), 1.92(\mathrm{~m}, 2 \mathrm{H}), 1.64-1.48(\mathrm{~m}, 4 \mathrm{H}) ;) ;{ }^{13} \mathrm{C}-\mathrm{NMR}\left(\mathrm{CDCl}_{3}, 125 \mathrm{MHz}\right) 206.3,151.0$, $145.2,129.2,128.9,127.2,125.5,125.2,121.6,81.6,69.1,55.4,45.8,41.3,28.8,25.9,23.4$. HRMS Calcd. For $\mathrm{C}_{23} \mathrm{H}_{26} \mathrm{NO}_{4}[\mathrm{M}+\mathrm{H}]: 380.1862$. Found: 380.1849 .

Preparation of Trans-phenyl 2-(2,3-dihydrobenzofuran-2-yl)-4-oxo-6-phenylpiperidine-1-carboxylate (26). According the the general procedure, reaction of $158 \mathrm{mg}(0.54 \mathrm{mmol})$ of dihydropyridone 5 with $177 \mathrm{mg}$ (1.08 mmol) of 2,3-dihydrobenzofuran-2-carboxylic acid 25 in the presence of $188 \mathrm{mg}(1.08 \mathrm{mmol})$ of $\mathrm{HK}_{2} \mathrm{PO}_{4}$ and $9.1 \mathrm{mg}(8.1 \mathrm{mmol})$ of $\left(\mathrm{Ir}\left[\mathrm{dF}\left(\mathrm{CF}_{3}\right)\right.\right.$ ppy $\left.] 2(\mathrm{dtbpy})\right) \mathrm{PF}_{6}$ provided $310 \mathrm{mg}(65 \%)$ of compound 26 as a separable 3:1 mixture of diastereomers and rotamers in $82 \%$ combined yield. Major isomer: colorless solid; ${ }^{1} \mathrm{H} \mathrm{NMR}\left(500 \mathrm{MHz}, \mathrm{CDCl}_{3}\right)$ $\delta 7.40(\mathrm{~m}, 4 \mathrm{H}), 7.35(\mathrm{~m}, 3 \mathrm{H}), 7.24(\mathrm{t}, J=7.1 \mathrm{~Hz}, 1 \mathrm{H}), 7.16(\mathrm{dd}, J=17.3,7.7 \mathrm{~Hz}, 2 \mathrm{H}), 7.04$ (br m, 2H), $6.89(\mathrm{t}, J=7.4 \mathrm{~Hz}, 1 \mathrm{H}), 6.74(\mathrm{~d}, J=8.0 \mathrm{~Hz}, 1 \mathrm{H}), 5.93(\mathrm{~d}, J=5.6 \mathrm{~Hz}, 1 \mathrm{H}), 5.49(\mathrm{t}$, $J=8.8 \mathrm{~Hz}, 1 \mathrm{H}), 4.70(\mathrm{~d}, J=6.9 \mathrm{~Hz}, 1 \mathrm{H}), 3.75(\mathrm{dd}, J=17.8,6.4 \mathrm{~Hz}, 1 \mathrm{H}), 3.52(\mathrm{dd}, J=16.3$, $10.2 \mathrm{~Hz}, 1 \mathrm{H}), 3.09(\mathrm{dd}, J=17.8,1.9 \mathrm{~Hz}, 1 \mathrm{H}), 2.95(\mathrm{dd}, J=16.3,7.6 \mathrm{~Hz}, 1 \mathrm{H}), 2.55(\mathrm{dd}, J=17.6$, $7.4 \mathrm{~Hz}, 1 \mathrm{H}), 2.41(\mathrm{~d}, J=17.6 \mathrm{~Hz}, 1 \mathrm{H}) .{ }^{13} \mathrm{C}-\mathrm{NMR}\left(\mathrm{CDCl}_{3}, 125 \mathrm{MHz}\right) \delta 205.3,159.0,150.9$, $129.4,129.3,129.1,129.0,128.6,128.3,127.5,127.4,125.8,125.6,125.3,125.2,124.9,121.6$, $121.4,121.3,121.2,109.5,58.0,55.4,45.5,44.7,36.1,33.1$. Anal. Calcd. For $\mathrm{C}_{26} \mathrm{H}_{23} \mathrm{NO}_{4}$ : C, 75.53; H, 5.61; N, 3.39. Found: C, 75.39; H, 5.59; N, 3.33. Minor isomer: colorless solid contaminated with some of the major isomer; ${ }^{1} \mathrm{H} \mathrm{NMR}\left(500 \mathrm{MHz}, \mathrm{CDCl}_{3}\right) \delta 7.40(\mathrm{~m}, 4 \mathrm{H})$, $7.35(\mathrm{~m}, 3 \mathrm{H}), 7.24(\mathrm{t}, J=7.1 \mathrm{~Hz}, 1 \mathrm{H}), 7.16(\mathrm{dd}, J=17.3,7.7 \mathrm{~Hz}, 2 \mathrm{H}), 7.04(\mathrm{br} \mathrm{m}, 2 \mathrm{H}), 6.94(\mathrm{~d}$, $J=7.4 \mathrm{~Hz}, 1 \mathrm{H}), 6.82(\mathrm{~d}, J=8.0 \mathrm{~Hz}, 2 \mathrm{H}), 5.70(\mathrm{~d}, J=6.5 \mathrm{~Hz}, 1 \mathrm{H}), 5.11(\mathrm{t}, J=5.1 \mathrm{~Hz}, 1 \mathrm{H}), 4.95$ $(\mathrm{td}, J=8.9,4.8 \mathrm{~Hz}, 1 \mathrm{H}), 3.51(\mathrm{~d}, J=10.2 \mathrm{~Hz}, 2 \mathrm{H}), 3.35(\mathrm{~d}, J=8.6 \mathrm{~Hz}, 2 \mathrm{H}), 2.79(\mathrm{~d}, J=18.0 \mathrm{~Hz}$, $1 \mathrm{H}) ;{ }^{13} \mathrm{C}-\mathrm{NMR}\left(\mathrm{CDCl}_{3}, 125 \mathrm{MHz}\right) \delta 205.1,204.4,159.1,158.4,150.9,150.8,141.6,129.7,129.4$, $129.3,129.1,129.0,128.6,127.5,127.4,126.5,125.8,125.7,125.3,125.2,125.1,125.0,124.9$, 121.7, 121.5, 121.3, 121.2, 109.5, 86.0, 84.0, 58.1, 55.4, 54.3, 36.1, 33.1. HRMS Calcd. For $\mathrm{C}_{26} \mathrm{H}_{24} \mathrm{NO}_{4}[\mathrm{M}+\mathrm{H}]: 414.4810$. Found: 414.4799 .

Preparation of Trans-phenyl-4-oxo-2-phenyl-6-tetrahydrofuran-2-yl)piperidine-1-carboxylate (28). According the the general procedure, reaction of $150 \mathrm{mg}(0.51 \mathrm{mmol})$ of dihydropyridone 5 with $119 \mathrm{mg}(1.02 \mathrm{mmol})$ tetrahydrofuran2-carboxylic acid 27 in the presence of $178 \mathrm{mg}$ (1.02 mmol) of $\mathrm{HK}_{2} \mathrm{PO}_{4}$ and $8.6 \mathrm{mg}$ (7.6 mmol) of ( $\mathrm{Ir}\left[\mathrm{dF}\left(\mathrm{CF}_{3}\right)\right.$ ppy]2(dtbpy)) $\mathrm{PF}_{6}$ provided $131 \mathrm{mg}(70 \%)$ of compound 28 as a separable 1:1 mixture of diastereomers: Major isomer: colorless oil; ${ }^{1} \mathrm{H}$ NMR $\left(500 \mathrm{MHz}, \mathrm{CDCl}_{3}\right) \delta 7.40-7.26(\mathrm{~m}, 7 \mathrm{H}), 7.21(\mathrm{~m}, 1 \mathrm{H}), 7.00(\mathrm{br} \mathrm{m}$, $2 \mathrm{H}), 5.83(\mathrm{~d}, 1 \mathrm{H}, J=5.6 \mathrm{~Hz}), 4.65(\mathrm{~d}, 1 \mathrm{H}, J=6.5 \mathrm{~Hz}), 4.57(\mathrm{t}, 1 \mathrm{H}, J=7.6 \mathrm{~Hz}), 3.73(\mathrm{~m}, 2 \mathrm{H})$, $3.67(\mathrm{dd}, 1 \mathrm{H}, J=17.8$ and $6.5 \mathrm{~Hz}), 3.00(\mathrm{dd}, 1 \mathrm{H}, J=17.8$ and $2.0 \mathrm{~Hz}), 2.53(\mathrm{dd}, 1 \mathrm{H}, J=17.4$ and $6.7 \mathrm{~Hz}), 2.45(\mathrm{~d}, 1 \mathrm{H}, J=16.8 \mathrm{~Hz}), 2.16(\mathrm{~m}, 1 \mathrm{H}), 1.90(\mathrm{~m}, 2 \mathrm{H}), 1.56(\mathrm{~m}, 1 \mathrm{H})$; ${ }^{13} \mathrm{C}-\mathrm{NMR}$ $\left(\mathrm{CDCl}_{3}, 125 \mathrm{MHz}\right) \delta 205.6,151.0,142.0,129.3,129.0,127.3,125.6,125.3,121.7,80.9,69.1,56.6$, 44.6, 36.8, 28.8, 25.8. HRMS Calcd. For $\mathrm{C}_{22} \mathrm{H}_{24} \mathrm{NO}_{4}[\mathrm{M}+\mathrm{H}]: 366.1705$. Found: 366.1699. Minor isomer: colorless oil; ${ }^{1} \mathrm{H}$ NMR $\left(500 \mathrm{MHz}, \mathrm{CDCl}_{3}\right) \delta 7.40-7.20(\mathrm{~m}, 7 \mathrm{H}), 7.17(\mathrm{~m}, 1 \mathrm{H})$, $6.86(\mathrm{br} \mathrm{m}, 2 \mathrm{H}), 5.76(\mathrm{~d}, 1 \mathrm{H}, J=6.2 \mathrm{~Hz}), 4.81(\mathrm{t}, 1 \mathrm{H}, J=6.2 \mathrm{~Hz}), 4.00-3.90(\mathrm{~m}, 2 \mathrm{H}), 3.82$ (q, 1H, J = 7.7 Hz), $3.48(\mathrm{~m}, 1 \mathrm{H}), 2.87(\mathrm{~m}, 2 \mathrm{H}), 2.60$ (d, 1H, J = 17.9 Hz), 2.08 (m, 2H), 1.95 $(\mathrm{m}, 1 \mathrm{H}), 1.72(\mathrm{~m}, 1 \mathrm{H}) ;{ }^{13} \mathrm{C}-\mathrm{NMR}\left(\mathrm{CDCl}_{3}, 125 \mathrm{MHz}\right) \delta 205.9,151.1,142.7,129.3,128.9,127.3$, $125.5,125.2,121.5,82.2,68.1,55.3,54.3,45.6,41.4,29.4,25.9$. HRMS Calcd. For $\mathrm{C}_{22} \mathrm{H}_{24} \mathrm{NO}_{4}$ $[\mathrm{M}+\mathrm{H}]:$ 366.1705. Found: 366.1712 . 
Preparation of Tert-butyl 2-(1-tert-butoxycarbonyl)azetidin-2-yl)-4-oxo-3,4-dihydroquinoline-1(2H)carboxylate (31). According the the general procedure, reaction of $159 \mathrm{mg}(0.65 \mathrm{mmol})$ of 4-oxoquinolinone 29 with $261 \mathrm{mg}(1.23 \mathrm{mmol})$ of $\mathrm{N}$-Boc-azetidine-2-carboxylic acid 13 in the presence of $182 \mathrm{mg}(1.23 \mathrm{mmol})$ of $\mathrm{HK}_{2} \mathrm{PO}_{4}$ and $10.9 \mathrm{mg}(9.72 \mathrm{mmol})$ of $\left(\mathrm{Ir}\left[\mathrm{dF}\left(\mathrm{CF}_{3}\right) \mathrm{ppy}\right] 2\right.$ (dtbpy)) $\mathrm{PF}_{6}$ provided $100 \mathrm{mg}(38 \%)$ of compound 31 as a separable mixture of diatereomers. Isomer A: ${ }^{1} \mathrm{H}$ NMR $\left(500 \mathrm{MHz}, \mathrm{CDCl}_{3}\right) \delta 7.98(\mathrm{dd}, J=7.8,1.4 \mathrm{~Hz}, 1 \mathrm{H}), 7.63(\mathrm{~d}, J=8.2 \mathrm{~Hz}, 1 \mathrm{H})$, $7.54-7.45(\mathrm{~m}, 1 \mathrm{H}), 7.17(\mathrm{t}, J=7.5 \mathrm{~Hz}, 1 \mathrm{H}), 5.12-5.01(\mathrm{~m}, 1 \mathrm{H}), 4.26-4.14(\mathrm{~m}, 1 \mathrm{H}), 3.96-3.78(\mathrm{~m}$, $2 \mathrm{H}), 3.19(\mathrm{~d}, J=18.3 \mathrm{~Hz}, 1 \mathrm{H}), 2.98(\mathrm{dd}, J=18.3,6.2 \mathrm{~Hz}, 1 \mathrm{H}), 2.26(\mathrm{p}, J=10.0,9.6 \mathrm{~Hz}, 1 \mathrm{H})$, $2.08(\mathrm{ddt}, J=11.3,8.8,5.7 \mathrm{~Hz}, 1 \mathrm{H}), 1.58(\mathrm{~s}, 9 \mathrm{H}), 1.42(\mathrm{~s}, 9 \mathrm{H}) .{ }^{13} \mathrm{C}-\mathrm{NMR}\left(\mathrm{CDCl}_{3}, 125 \mathrm{MHz}\right)$ $\delta$ 192.6, 157.1, 153.1, 141.6, 133.8, 126.8, 125.7, 124.8, 124.0, 61.7, 57.7, 46.9, 20.3. HRMS Calcd. For $\mathrm{C}_{22} \mathrm{H}_{31} \mathrm{~N}_{2} \mathrm{O}_{5}$ : 403.4990. Found: 403.4986. Isomer B: ${ }^{1} \mathrm{H}$ NMR (500 MHz, $\left.\mathrm{CDCl}_{3}\right) \delta 7.91$ $(\mathrm{d}, J=7.8 \mathrm{~Hz}, 1 \mathrm{H}), 7.78(\mathrm{~d}, J=8.3 \mathrm{~Hz}, 1 \mathrm{H}), 7.51(\mathrm{t}, J=7.8 \mathrm{~Hz}, 1 \mathrm{H}), 7.13(\mathrm{t}, J=7.5 \mathrm{~Hz}, 1 \mathrm{H})$, $5.09(\mathrm{t}, J=6.3 \mathrm{~Hz}, 1 \mathrm{H}), 4.39(\mathrm{dt}, J=8.6,5.6 \mathrm{~Hz}, 1 \mathrm{H}), 3.75-3.47(\mathrm{~m}, 2 \mathrm{H}), 3.12(\mathrm{dd}, J=18.3$, $7.4 \mathrm{~Hz}, 1 \mathrm{H}), 2.89(\mathrm{~d}, J=18.3 \mathrm{~Hz}, 1 \mathrm{H}), 2.28(\mathrm{~s}, 1 \mathrm{H}), 2.01(\mathrm{~d}, J=22.3 \mathrm{~Hz}, 1 \mathrm{H}), 1.57(\mathrm{~s}, 9 \mathrm{H}), 1.36$ $(\mathrm{s}, 9 \mathrm{H}) .{ }^{13} \mathrm{C}-\mathrm{NMR}\left(\mathrm{CDCl}_{3}, 125 \mathrm{MHz}\right) \delta 192.7,156.0,153.4,142.7,133.7,126.3,125.0,123.6$, 82.1, 56.1, 46.8, 40.0, 28.4, 28.2, 19.4. HRMS Calcd. For $\mathrm{C}_{22} \mathrm{H}_{31} \mathrm{~N}_{2} \mathrm{O}_{5}[\mathrm{M}+\mathrm{H}]: 403.4990$. Found: 403.4999 .

Preparation of Tert-butyl 2-(1-(tert-butoxycarbonyl)pyrrolidin-2-yl)-4-oxo-3,4-dihydroquinoline1(2H) Carboxylate (32). According the the general procedure, reaction of $128 \mathrm{mg}(0.52 \mathrm{mmol})$ of 4-oxoquinolinone 29 with $225 \mathrm{mg}(1.04 \mathrm{mmol})$ of $\mathrm{N}$-Boc-L-proline 11 in the presence of $182 \mathrm{mg}(1.04 \mathrm{mmol})$ of $\mathrm{HK}_{2} \mathrm{PO}_{4}$ and $8.8 \mathrm{mg}(7.83 \mathrm{mmol})$ of $\left(\mathrm{Ir}\left[\mathrm{dF}\left(\mathrm{CF}_{3}\right)\right.\right.$ ppy $\left.2(\mathrm{dtbpy})\right) \mathrm{PF}_{6}$ provided $120 \mathrm{mg}$ (55\%) of compound 32 as a colorless oil and an inseparable 1:1 mixture of diastereomers and rotamers: ${ }^{1} \mathrm{H}$ NMR $\left(500 \mathrm{MHz}, \mathrm{CDCl}_{3}\right) \delta 8.05-7.71(\mathrm{br} \mathrm{m}, 1 \mathrm{H}), 7.78,7.60$ and $7.5(\mathrm{~m}, 2 \mathrm{H}), 7.20-7.10(\mathrm{~m}, 1 \mathrm{H}), 4.87$ and $4.60(\mathrm{~m}, 1 \mathrm{H}), 4.15-3.93(\mathrm{~m}, 1.90 \mathrm{H}), 3.04(\mathrm{dd}$, $0.26 \mathrm{H}, J=17.8$ and $6.9 \mathrm{~Hz}), 2.94-2.89(\mathrm{~m}, 2 \mathrm{H}), 2.05-1.85(\mathrm{br} \mathrm{m}, 3.7 \mathrm{H}), 1.68(\mathrm{~m}, 1 \mathrm{H}), 1.56$ and 1.55 (s, 9H), 1.35 (br m, 9H); ${ }^{13} \mathrm{C}-\mathrm{NMR}\left(\mathrm{CDCl}_{3}, 125 \mathrm{MHz}\right) \delta$ 192.8, 192.7, 155.1, 154.6, 153.4, $153.2,143.7,141.3,133.8,133.5,132.8,127.0,126.3,126.2,125.2,125.1,124.2,123.2,82.4,81.0$, 59.1, 57.8, 57.2, 56.6, 53.9, 46.8, 46.2, 45.9, 40.9, 40.8, 40.2, 39.7, 28.4, 28.3, 28.2, 28.0, 23.8, 23.5, 22.7, 22.3. HRMS Calcd. For $\mathrm{C}_{23} \mathrm{H}_{33} \mathrm{~N}_{2} \mathrm{O}_{5}[\mathrm{M}+\mathrm{H}]:$ : 417.5260. Found: 417.5251.

Preparation of Tert-butyl (2-(1-(tert-butoxycarbonyl)piperidin-2-yl)-4-oxo-3,4-dihydroquinoline$1(2 \mathrm{H})$-carboxylate (33). According the the general procedure, reaction of $164 \mathrm{mg}(0.67 \mathrm{mmol})$ of 4-oxoquinolinone 29 with $310 \mathrm{mg}(1.37 \mathrm{mmol})$ of $N$-Boc-pipecolic acid 14 in the presence of $233 \mathrm{mg}(1.37 \mathrm{mmol})$ of $\mathrm{HK}_{2} \mathrm{PO}_{4}$ and $11 \mathrm{mg}(10.0 \mathrm{mmol})$ of $\left(\mathrm{Ir}\left[\mathrm{dF}\left(\mathrm{CF}_{3}\right)\right.\right.$ ppy $] 2(\mathrm{dtbpy}) \mathrm{PF}_{6}$ provided $160 \mathrm{mg}$ (55\%) of compound 33 as a colorless oil and as inseparable 4:1 mixture of diastereomers and rotamers: ${ }^{1} \mathrm{H}$ NMR $\left(500 \mathrm{MHz}, \mathrm{CDCl}_{3}\right) \delta 8.03$ and $8.97(\mathrm{~d}, 1 \mathrm{H}, \mathrm{J}=7.8 \mathrm{~Hz})$, $7.71-7.45(\mathrm{~m}, 2 \mathrm{H}), 7.20(\mathrm{~m}, 1 \mathrm{H}), 5.36$ and $5.24(\mathrm{~m}, 1 \mathrm{H}), 4.36$ and $4.21(\mathrm{~m}, 1 \mathrm{H}), 4.13(\mathrm{~m}, 0.8 \mathrm{H})$, 4.01-3.80 (m, 0.4H), 3.14-3.01 (m, 0.7H), $2.91(\mathrm{dd}, 0.8 \mathrm{H}, J=18.3$ and $6.0 \mathrm{~Hz}), 2.78-2.57(\mathrm{~m}$, $1.8 \mathrm{H}), 1.84-1.64(\mathrm{~m}, 5 \mathrm{H}), 1.51(\mathrm{~s}, 10.8 \mathrm{H}), 1.44-1.35(\mathrm{~m}, 2 \mathrm{H}), 1.33(\mathrm{~s}, 6 \mathrm{H}) ;{ }^{13} \mathrm{C}-\mathrm{NMR}\left(\mathrm{CDCl}_{3}\right.$, $125 \mathrm{MHz}) \delta$ 192.9, 192.5, 192.1, 154.4, 152.9, 142.1, 141.1, 140.8, 134.3, 133.8, 133.5, 127.2, $126.9,126.5,126.2,125.9,125.8,125.5,125.2,124.5,124.3,123.8,82.4,80.6,51.5,50.7,50.3$, $50.1,48.8,48.7,40.7,40.4,39.9,39.4,38.7,28.5,28.3,27.9,26.0,25.2,25.1,24.7,19.2$. HRMS Calcd. For $\mathrm{C}_{24} \mathrm{H}_{35} \mathrm{~N}_{2} \mathrm{O}_{5}[\mathrm{M}+\mathrm{H}]:$ 431.5530. Found: 431.5528 .

Preparation of Tert-butyl-3-(1-(tert-butoxycarbonyl)-4-oxo-1,2,3,4-tetrahydroquinolin-2-yl) morpholine-4-carboxylate (34). According the (the general procedure, reaction of $106 \mathrm{mg}$ $(0.43 \mathrm{mmol})$ of 4-oxoquinolinone 29 with $200 \mathrm{mg}(0.87 \mathrm{mmol})$ of 4-Boc-3-morpholinecarboxylic acid 16 in the presence of $151 \mathrm{mg}(0.87 \mathrm{mmol})$ of $\mathrm{HK}_{2} \mathrm{PO}_{4}$ and $7.3 \mathrm{mg}(6.5 \mathrm{mmol})$ of $\left(\operatorname{Ir}\left[\mathrm{dF}\left(\mathrm{CF}_{3}\right)\right.\right.$ ppy $\left.] 2(\mathrm{dtbpy})\right) \mathrm{PF}_{6}$ provided $122 \mathrm{mg}(65 \%)$ of compound 34 as a colorless foam and as inseparable 4:1 mixture of diastereomers and rotamers: ${ }^{1} \mathrm{H} \mathrm{NMR}\left(500 \mathrm{MHz}, \mathrm{CDCl}_{3}\right)$ $\delta 8.01$ and $8.00(\mathrm{~m}, 1 \mathrm{H}), 7.79-7.60(\mathrm{~m}, 1 \mathrm{H}), 7.54(\mathrm{~m}, 1 \mathrm{H}), 7.20(\mathrm{~m}, 1 \mathrm{H}), 5.57$ and $5.50(\mathrm{~m}$, $1 \mathrm{H}), 4.10$ and $4.02(\mathrm{~m}, 1.2 \mathrm{H}), 3.94-3.74(\mathrm{~m}, 3 \mathrm{H}), 3.57-3.34(\mathrm{~m}, 2.5 \mathrm{H}), 3.15(\mathrm{dd}, 0.5 \mathrm{H}, J=17.9$ and $5.5 \mathrm{~Hz}), 3.07-2.93(\mathrm{~m}, 1.3 \mathrm{H}), 2.82(\mathrm{t}, 0.3 \mathrm{H}, J=17.7$ and $5.5 \mathrm{~Hz}), 2.69$ and $2.55(\mathrm{~d}, 0.51 \mathrm{H}$, $J=18.3 \mathrm{~Hz}), 1.59(\mathrm{~m}, 9.5 \mathrm{H}), 1.46$ and $1.41(\mathrm{~s}, 4 \mathrm{H}), 1.36(\mathrm{~s}, 5 \mathrm{H}), 1.10(\mathrm{~s}, 1.8 \mathrm{~Hz}) ;{ }^{13} \mathrm{C}-\mathrm{NMR}$ $\left(\mathrm{CDCl}_{3}, 125 \mathrm{MHz}\right) \delta 192.7,192.1,191.6,154.1,154.0,152.9,152.8,142.2,141.2,140.9,134.5$, 
134.0, 133.6, 127.3, 127.1, 126.6, 126.2, 125.7, 125.5, 125.3, 125.1, 124.5, 124.2, 124.0, 123.9, 82.9, 82.7, 82.4, 81.1, 67.4, 67.0, 66.9, 66.7, 66.4, 66.0, 65.8, 51.5, 50.9, 50.2, 49.6, 49.3, 40.6, 40.2, 38.9, 38.7, 28.3, 28.1, 27.9. HRMS Calcd. For $\mathrm{C}_{23} \mathrm{H}_{33} \mathrm{~N}_{2} \mathrm{O}_{5}[\mathrm{M}+\mathrm{H}]: 433.5250$. Found: 433.5243.

Preparation of Tert-butyl 2-(4-oxochroman-2-yl)pyrrolidine-1-carboxylate (35). According the the general procedure, reaction of $157 \mathrm{mg}(1.07 \mathrm{mmol})$ of $4 H$-chromen-4-one 30 with $462 \mathrm{mg}$ $(2.15 \mathrm{mmol})$ of $\mathrm{N}$-Boc-L-proline 11 in the presence of $374 \mathrm{mg}(2.15 \mathrm{mmol})$ of $\mathrm{HK}_{2} \mathrm{PO}_{4}$ and $18 \mathrm{mg}(0.016 \mathrm{mmol})$ of $\left(\operatorname{Ir}\left[\mathrm{dF}\left(\mathrm{CF}_{3}\right) \mathrm{ppy}\right] 2(\mathrm{dtbpy})\right) \mathrm{PF}_{6}$ provided $310 \mathrm{mg}(65 \%)$ of compound 35 as a colorless foam and as inseparable 1:1 mixture of diastereomers and rotamers: ${ }^{1} \mathrm{H}$ $\operatorname{NMR}\left(500 \mathrm{MHz}, \mathrm{CDCl}_{3}\right) \delta 7.90(\mathrm{~m}, 1 \mathrm{H}), 7.49$ (m, 1H), 7.00 (br m, 2H), 4.85-4.54 (br m, 1H), 4.27 (br m, 0.5H), 4.05 (br m, 0.5H), 3.69-3.35 (br m, 2H), 2.78 (m, 1H), 2.67 (m, 1H), 2.25 (m, 0.5H), 2.14-1.88 (br m, 3.5H), 1.48 (s, 9H); ${ }^{13} \mathrm{C}-\mathrm{NMR}\left(\mathrm{CDCl}_{3}, 125 \mathrm{MHz}\right) \delta 192.5,192.0,161.5$, 156.2, 155.0, 135.8, 126.9, 126.8, 121.2, 120.9, 118.0, 117.8, 80.1, 79.5, 78.9, 78.4, 77.9, 59.8, 58.8, 47.3, 47.1, 46.7, 42.0, 39.9, 39.4, 28.5, 27.7, 26.6, 25.7, 24.5, 24.2, 23.6, 23.3. HRMS Calcd. For $\mathrm{C}_{18} \mathrm{H}_{24} \mathrm{NO}_{4}[\mathrm{M}+\mathrm{H}]: 317.1627$. Found: 317.1624.

Preparation of Tert-butyl-2-(4-oxochroman-2-yl)indoline-1-carboxylate (36). According the the general procedure, reaction of $157 \mathrm{mg}(1.07 \mathrm{mmol})$ of $4 H$-chromen-4-one 30 with $424 \mathrm{mg}$ (2.15 mmol) of $\mathrm{N}$-Boc-indoline-2-carboxylic acid 21 in the presence of $374 \mathrm{mg}(2.15 \mathrm{mmol})$ of $\mathrm{HK}_{2} \mathrm{PO}_{4}$ and $18 \mathrm{mg}(0.016 \mathrm{mmol})$ of $\operatorname{Ir}\left[\mathrm{dF}\left(\mathrm{CF}_{3}\right)\right.$ ppy]2(dtbpy) $) \mathrm{PF}_{6}$ provided $240 \mathrm{mg}(61 \%)$ of compound 36 as a colorless oil as an inseparable 1:1 mixture of diastereomers and rotamers: ${ }^{1} \mathrm{H}$ NMR $\left(500 \mathrm{MHz}, \mathrm{CDCl}_{3}\right) \delta 7.87(\mathrm{~m}, 1 \mathrm{H}), 7.48(\mathrm{~m}, 1 \mathrm{H}), 7.20(\mathrm{~m}, 2 \mathrm{H}), 7.05-6.89(\mathrm{~m}, 3 \mathrm{H})$, 4.95 (br m, 1H), 4.74 and $4.70($ br m, 1H), $3.42(\mathrm{~m}, 1 \mathrm{H}), 3.34-3.23(\mathrm{~m}, 1 \mathrm{H}), 2.90(\mathrm{dd}, J=15.0$, $10.0 \mathrm{~Hz}, 0.5 \mathrm{H}), 2.72(\mathrm{dd}, J=15.0,5.0 \mathrm{~Hz}, 0.5 \mathrm{H}), 2.62(\mathrm{dd}, J=15.0$ and $14.0 \mathrm{~Hz}, 0.5 \mathrm{H}), 2.49$ $(\mathrm{dd}, J=15.0,5.0 \mathrm{~Hz}, 0.5 \mathrm{H}), 1.60(\mathrm{~s}, 9 \mathrm{H}) .{ }^{13} \mathrm{C}-\mathrm{NMR}\left(\mathrm{CDCl}_{3}, 125 \mathrm{MHz}\right) \delta 191.9,191.5,161.4$, 161.3, 136.0, 135.8, 127.7, 127.4, 127.0, 126.9, 124.7, 124.5, 123.1, 123.0, 121.5, 121.1, 120.9, $118.1,117.9,115.9,115.7,78.1,77.1,61.5,60.3,39.9,37.1,28.4$. HRMS Calcd. For $\mathrm{C}_{22} \mathrm{H}_{24} \mathrm{NO}_{4}$ $[\mathrm{M}+\mathrm{H}]: 366.4370$. Found: 366.4366 .

Preparation of 2-(Tetrahydrofuran-2-yl)chroman-4-one (37). According the the general procedure, reaction of $133 \mathrm{mg}(0.91 \mathrm{mmol})$ of $4 \mathrm{H}$-chromen-4-one 30 with $211 \mathrm{mg}(1.82 \mathrm{mmol})$ of tetrahydrofuran2-carboxylic acid 27 in the presence of $317 \mathrm{mg}(1.82 \mathrm{mmol})$ of $\mathrm{HK}_{2} \mathrm{PO}_{4}$ and $15 \mathrm{mg}(0.14 \mathrm{mmol})$ of $\left(\operatorname{Ir}\left[\mathrm{dF}\left(\mathrm{CF}_{3}\right)\right.\right.$ ppy $\left.] 2(\mathrm{dtbpy})\right) \mathrm{PF}_{6}$ provided $135 \mathrm{mg}$ (68\%) of compound 37 as a separable 1:1 mixture of diastereomers and as colorless oils: Isomer 1: ${ }^{1} \mathrm{H}$ NMR $\left(500 \mathrm{MHz} \mathrm{CDCl}_{3}\right) \delta 7.90(\mathrm{dd}, J=7.8,1.3 \mathrm{~Hz}, 1 \mathrm{H}), 7.54-7.44(\mathrm{~m}, 1 \mathrm{H}), 7.07-6.97(\mathrm{~m}, 2 \mathrm{H}), 4.41$ $(\mathrm{dt}, J=11.9,4.5 \mathrm{~Hz}, 1 \mathrm{H}), 4.20(\mathrm{q}, J=6.7 \mathrm{~Hz}, 1 \mathrm{H}), 3.95(\mathrm{q}, J=6.7 \mathrm{~Hz}, 1 \mathrm{H}), 3.86(\mathrm{q}, J=6.8 \mathrm{~Hz}$, $1 \mathrm{H}), 2.90-2.72(\mathrm{~m}, 2 \mathrm{H}), 2.21-2.07(\mathrm{~m}, 1 \mathrm{H}), 1.95(\mathrm{M}, 3 \mathrm{H}) .{ }^{13} \mathrm{C}-\mathrm{NMR}\left(\mathrm{CDCl}_{3}, 125 \mathrm{MHz}\right) \delta 192.2$, 161.4, 136.0, 126.9, 121.4, 121.1, 118.0, 79.7, 79.4, 69.0, 39.0, 27.6, 25.7. Isomer 2: ${ }^{1} \mathrm{H}$ NMR $\left(500 \mathrm{MHz}_{\mathrm{CDCl}}\right) \delta 7.90(\mathrm{~d}, J=7.8 \mathrm{~Hz}, 1 \mathrm{H}), 7.50(\mathrm{t}, J=7.8 \mathrm{~Hz}, 1 \mathrm{H}), 7.14-6.97(\mathrm{~m}, 2 \mathrm{H}), 4.43$ $(\mathrm{ddd}, J=13.1,5.0,2.9 \mathrm{~Hz}, 1 \mathrm{H}), 4.14(\mathrm{q}, J=7.0 \mathrm{~Hz}, 1 \mathrm{H}), 4.03-3.72(\mathrm{~m}, 2 \mathrm{H}), 2.93(\mathrm{dd}, J=16.7$, $13.2 \mathrm{~Hz}, 1 \mathrm{H}), 2.68(\mathrm{dd}, J=16.7,2.8 \mathrm{~Hz}, 1 \mathrm{H}), 2.15-1.83(\mathrm{~m}, 4 \mathrm{H}) .{ }^{13} \mathrm{C}-\mathrm{NMR}\left(\mathrm{CDCl}_{3}, 125 \mathrm{MHz}\right)$ $\delta 192.1,161.3,136.1,126.8,121.3,120.9,118.1,79.8,79.7,68.9,39.8,27.5,25.9$.

Preparation of tert-Butyl 2-(4-oxochroman-2-yl)piperidine-1-carboxylate (38). According the the general procedure, reaction of $239 \mathrm{mg}(1.64 \mathrm{mmol})$ of $4 \mathrm{H}$-chromen-4-one 30 with $750 \mathrm{mg}$ ( $3.27 \mathrm{mmol})$ of $N$-Boc-pipecolic acid 15 in the presence of $570 \mathrm{mg}(3.27 \mathrm{mmol})$ of $\mathrm{HK}_{2} \mathrm{PO}_{4}$ and $18.3 \mathrm{mg}(0.016 \mathrm{mmol})$ of $\left(\mathrm{Ir}\left[\mathrm{dF}\left(\mathrm{CF}_{3}\right)\right.\right.$ ppy]2(dtbpy) $) \mathrm{PF}_{6}$ provided $500 \mathrm{mg}(92 \%)$ of compound 38 as a separable 1:1 mixture of diastereomers and rotamers: Isomer \#1: colorless solid; ${ }^{1} \mathrm{H}$ NMR $\left(500 \mathrm{MHz}, \mathrm{CDCl}_{3}\right) \delta 7.92(\mathrm{dd}, 1 \mathrm{H}, J=7.18$ and $1.6 \mathrm{~Hz}), 7.50(\mathrm{~m}$, $1 \mathrm{H}), 7.05(\mathrm{t}, 1 \mathrm{H}, J=7.5 \mathrm{~Hz}), 7.01(\mathrm{~d}, 1 \mathrm{H}, J=8.3 \mathrm{~Hz}), 4.74(\mathrm{~m}, 1 \mathrm{H}), 4.50(\mathrm{br} \mathrm{m}, 1 \mathrm{H}), 4.14(\mathrm{br} \mathrm{m}$, $1 \mathrm{H}), 2.75(\mathrm{~m}, 3 \mathrm{H}), 2.23(\mathrm{~m}, 1 \mathrm{H}), 1.75-1.60(\mathrm{~m}, 5 \mathrm{H}), 1.49(\mathrm{~s}, 9 \mathrm{H}) ;{ }^{13} \mathrm{C}-\mathrm{NMR}\left(\mathrm{CDCl}_{3}, 125 \mathrm{MHz}\right)$ $\delta 191.9,161.1,154.8,135.9,127.0,121.5,121.2,117.9,80.1,74.8,52.7,39.9,28.4,25.1,24.2,19.2$. Anal. Cald. For $\mathrm{C}_{19} \mathrm{H}_{25} \mathrm{NO}_{4}$ : C, 68.86; H, 7.60; N, 4.23. Found: C, 68.96; H, 7.55; N, 4.19. Isomer \#2: colorless oil; ${ }^{1} \mathrm{H}$ NMR $\left(500 \mathrm{MHz} \mathrm{CDCl}_{3}\right) \delta 7.90(\mathrm{dd}, 1 \mathrm{H}, J=7.9$ and $1.6 \mathrm{~Hz}), 7.49$ $(\mathrm{m}, 1 \mathrm{H}), 7.04(\mathrm{~m}, 1 \mathrm{H}), 6.97(\mathrm{~d}, 1 \mathrm{H}, J=8.3 \mathrm{~Hz}), 4.73(\mathrm{q}, 1 \mathrm{H}, J=7.7 \mathrm{~Hz}), 4.50($ br m, 1H) 4.15 (br m, 1H), 2.96 (br m, 1H), 2.77 (m, 2H), 1.78 (m, 2H), 1.70 (m, 3H), 1.53 (m, 1H), 1.49 (s, 
$9 \mathrm{H}) ;{ }^{13} \mathrm{C}-\mathrm{NMR}\left(\mathrm{CDCl}_{3}, 125 \mathrm{MHz}\right) \delta$ 192.0, 161.3, 155.5, 136.0, 126.8, 121.4, 120.9, 118.1, 79.6, 53.0, 40.7, 39.9, 28.4, 25.8, 25.0, 19.8. Anal.Calcd. for $\mathrm{C}_{19} \mathrm{H}_{26} \mathrm{NO}_{4}[\mathrm{M}+\mathrm{H}]: 332.1862$. Found: 332.1870 .

\section{Conclusions}

In conclusion, we have demonstrated that the photoredox-catalyzed decarboxylative formation of carbon-centered radicals from cyclic amino acids followed by conjugate addition to both cyclic vinylogous amides and esters provides access to novel heterocyclic structures. This versatile method is both mild and efficient giving rise to structural complexity previously inaccessible through current synthetic methodologies. Further manipulation of the products toward more complex synthetic targets is possible and will be disclosed in due course.

Supplementary Materials: The following supporting information can be downloaded. Full characterization $\left({ }^{1} \mathrm{H}\right.$ NMR and ${ }^{13} \mathrm{C}$ NMR spectra) of all new compounds.

Author Contributions: Conceptualization and experimental execution, J.T.K.; NMR analysis, A.B. and Q.G.; Reaction screening, K.D.; modeling, Y.-H.L. All authors have read and agreed to the published version of the manuscript.

Funding: This research received no external funding.

Institutional Review Board Statement: Not applicable.

Informed Consent Statement: Not applicable.

Data Availability Statement: Data are available in Electronic Supporting Information (ESI), and for additional details, please contact the authors.

Acknowledgments: The Authors thank David Thaisrivongs, Artis Klapars, Marc R. Becker, and Shorouk Badir of Merck \& Co., Inc., Kenilworth, NJ, USA for helpful discussions during the preparation of this manuscript.

Conflicts of Interest: The authors declare no conflict of interest.

Sample Availability: Not applicable.

\section{References}

1. Geise, B.; Dupuis, J. Diastereoselective Synthesis of C-Glycopyranosides. Angew. Chem. Int. Ed. Engl. 1983, 22, 622-623. [CrossRef]

2. Giese, B. Formation of CC Bonds by Addition f Free Radicals to Alkenes. Angew. Chem. Int. Ed. Engl. 1983, 22, 753-764. [CrossRef]

3. Giese, B.; Gonzáles-Gómez, J.A. The Scope of Radical CC-Coupling by the "Tin Method". Angew. Chem. Int. Ed. Engl. 1984, 23, 69-70. [CrossRef]

4. Jasperse, C.P., D.P.; Curran, D.P.; Fevig, T.L. Radical Reactions in Natural Product Synthesis. Chem. Rev. 1991, 91, 1237-1286. [CrossRef]

5. Zhang, W. Intramolecular Free Radical Conjugate Additions. Tetrahedron 2001, 57, 7237-7262. [CrossRef]

6. Srikanth, G.S.C.; Castle, S.L. Advances in Radical Conjugate Additions. Tetrahedron 2005, 61, 10377-10441. [CrossRef]

7. Rowlands, G.J. Radicals in Organic Synthesis: Part 2. Tetrahedron 2010, 66, 1593-1636. [CrossRef]

8. Chu, L.; Ohta, Z.Z.; MacMillan, D.W.C. Carboxylic Acids as a Traceless Activation Group for Conjugate Additions: A Three-step Synthesis of ( \pm )-Pregabalin. J. Am. Chem. Soc. 2014, 136, 10886-10889. [CrossRef] [PubMed]

9. Millet, A.; Lefebvre, Q.; Rueping, M. Visible-Light Photoredox-Catalyzed Giese Reaction: Decarboxylative Addition of Amino Acid Derived $\alpha$-Amino Radicals to Electron-Deficient Olefins. Chem. Eur. J. 2016, 22, 13464-13468. [CrossRef]

10. Gualandi, A.; Matteucci, E.; Monti, F.; Baschieri, A.; Armaroli, N.; Sambri, L.; Cozzi, P.G. Photoredox Radical Conjugate Addition of Dithiane-2-carboxylate Promoted by an Iridium(III)phenyl-tertrazole complex: A Formal Radical Methylation to Michael Acceptors. Chem. Sci. 2017, 8, 1613-1620. [CrossRef] [PubMed]

11. Li, Y.; Miyazawa, K.; Koike, T.; Akita, M. Alkyl- and Aryl-Thioalkylation of Olefins with Groganoftrifluoroborates by Photoredox Catalysis. Org. Chem. Front. 2015, 2, 319-323. [CrossRef]

12. Miyazawa, K.; Yasu, Y.; Koike, T.; Akita, M. Visible-Light-Induced Hydroalkoxymethylation of Electron Deficient Alkenes by Photoredox Catalysis. Chem. Commun. 2013, 49, 7249-7251. [CrossRef]

13. Nawrat, C.; Jamison, C.R.; Slutskyy, Y.; MacMillan, D.W.C.; Overman, L.E. Oxylates as Activating Groups for Alcohols in Visible Light Photoredox Catalysis: Formation of Quaternary Centers by Redox-Neutral Fragment Coupling. J. Am. Chem. Soc. 2015, 137, 11270-11273. [CrossRef] 
14. lacker, G.L.; Quasdorf, K.W.; Overman, L.E. Direct Construction of Quaternary Carbons from Tertiary Alcohols via PhotoredoxCatalyzed Fragmentation of tert-Alkyl N-phthalimidoyl Oxalates. J. Am. Chem. Soc. 2013, 135, 15342-15345. [CrossRef]

15. Slutskyy, Y.; Overman, L.E. Generation of Methoxycarbonyl Radical by Visible-Light Photoredox Catalysis and its Conjugate Addition with Electron-Deficient Olefins. Org. Lett. 2016, 18, 2564-2567. [CrossRef]

16. Corcé, V.; Chamoreau, L.-M.; Derat, E.; Goddard, J.-P.; Ollivier, C.; Fensterbank, L. Silicates as Latent Alkyl Radical Precursors: Visible-Light Photocatalytic Oxidation of Hypervalent Bis-catecholato Silicon Compunds. Angew. Chem. Int. Ed. Engl. 2015, 54, 11414-11418. [CrossRef]

17. Jouffroy, M.; Primer, D.N.; Molander, G.A. Base-free Photoredox/Nickel Dual-Catalytic Cross-Coupling of Ammonium Alkylsilicates. J. Am. Chem. Soc. 2016, 138, 475-478. [CrossRef]

18. Raynor, K.D.; May, G.D.; Bandarage, U.K.; Boyd, M.J. Generation of Diversity Sets with High sp ${ }^{3}$ Fraction using the Photoredox Coupling of Organotrifluoroborates and Organosilicates with Heteroaryl/aryl Bromides in Continuous Flow. J. Org. Chem. 2018, 83, 1551-1557. [CrossRef]

19. ElMarrouni, A.; Ritts, C.B.; Balsells, J. Silyl-Mediated Photoredox-Catalyzed Giese Reaction: Addition of Non-activated Alkyl Bromides. Chem. Sci. 2018, 9, 6639-6646. [CrossRef]

20. Lee, G.S.; hong, S.H. Formal Giese addition of C( $\left.\mathrm{sp}^{3}\right)-\mathrm{H}$ Nucleophiles Enabled by Visible Light Mediated Ni Catalysis of Triplet Enone Diradicals. Chem. Sci. 2018, 9, 5810-5815. [CrossRef]

21. Comins, D.L.; Brown, J.D. Addition of Grignard Reagents to 1-Acyl-4-methoxypyridinum Salt. An Approach to the Synthesis of Quinolizidinones. Tet. Lett. 1986, 27, 4549-4552.

22. Kelly, C.B.; Patel, N.R.; Primer, D.N.; Jouffroy, M.; Tellis, J.C.; Molander, G.A. Preparation of Visible-light-Activated Metal Complexes and Their Use in Photoredox/Nickel Dual Catalysis. Nat. Protoc. 2017, 12, 472-492. [CrossRef]

23. Shang, T.-Y.; Lu, L.-H.; Cao, Z.; Liu, Y.; He, W.-M.; Yu, B. Recent Advances of 1,2,3,5-Tetrakis(carbazol-9-yl)-4,6-dicyanobenzene (4CzIPN) in Photocatalytic Transformations. Chem. Commun. 2019, 55, 5408-5419. [CrossRef]

24. Rosi, F.; Crucitti, G.C.; Iacovo, A.; Miele, G.; Pescatori, L.; Santo, R.D.; Costi, R. Convient Route to 2H-Pyrrolo[3,4- $b$ ]quinolin9(4H)-one Skeleton via Tosmic Reaciton. Syn. Commun. 2013, 43, 1063-1072. [CrossRef] 